High Molecular Orientation in Mono- and Tri-layer Polydiacetylene Films Imaged by Atomic Force Microscopy

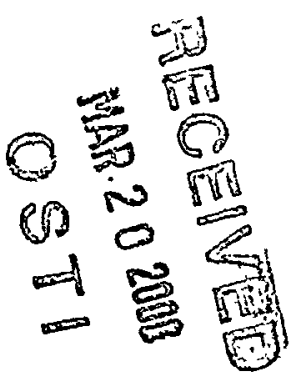

Darryl Y. Sasaki*, Robert W. Carpick, and Alan R. Burns

Sandia National Laboratories

Biomolecular Materials and Interface Science Department

Albuquerque, NM 87185-1413

\author{
Abbreviated title: \\ Molecular Orientation in Polydiacetylene Films
}

To whom correspondence should be sent:

Darryl Y. Sasaki, Ph.D.

Sandia National Laboratories

Biomolecular Materials and Interface Science Department

Albuquerque, NM 87185-1413 


\section{DISCLAIMER}

This report was prepared as an account of work sponsored by an agency of the United States Government. Neither the United States Government nor any agency thereof, nor any of their employees, make any warranty, express or implied, or assumes any legal liability or responsibility for the accuracy, completeness, or usefulness of any information, apparatus, product, or process disclosed, or represents that its use would not infringe privately owned rights. Reference herein to any specific commercial product, process, or service by trade name, trademark, manufacturer, or otherwise does not necessarily constitute or imply its endorsement, recommendation, or favoring by the United States Government or any agency thereof. The views and opinions of authors expressed herein do not necessarily state or reflect those of the United States Government or any agency thereof. 


\section{DISCLAIMER}

Portions of this document may be illegible in electronic image products. Images are produced from the best available original document. 


\title{
High Molecular Orientation in Mono- and Tri-layer Polydiacetylene Films Imaged by Atomic Force Microscopy
}

\author{
Darryl Y. Sasaki*, Robert W. Carpick ${ }^{\S}$, and Alan R. Burns \\ Sandia National Laboratories \\ Biomolecular Materials and Interfaces Department \\ Albuquerque, NM 87185-1413
}

\begin{abstract}
Atomically flat monolayer and trilayer films of polydiacetylenes have been prepared on mica and silicon using a horizontal deposition technique from a pure water subphase. Langmuir films of 10,12-pentacosadiynoic acid (I) and N-(2-ethanol)-10,12pentacosadiynamide (II) were compressed to $20 \mathrm{mN} / \mathrm{m}$ and subsequently polymerized by UV irradiation at the air-water interface. Blue and red forms of the films were prepared by varying exposure times and incident power. Polymerization to the blue-phase films produced slight contractions in the film of 2 and $5 \%$ for the films of II and I, respectively. Longer UV exposures yielded red-phase films with dramatic film contraction of 15 and $32 \%$ for II and I, respectively. The horizontal deposition technique provided transfer ratios of unity with minimal film stress or structure modification.

Atomic force microscopy images revealed nearly complete coverage of the substrate with atomically flat films. Crystalline domains of up to 100 microns of highly oriented polydiacetylene molecules were observed. The results reported herein provided insight into the roles of molecular packing and chain orientations in converting the monomeric film to the polymerized blue- and red-phases.
\end{abstract}

Keywords: Polydiacetylene, Langmuir films, AFM, horizontal deposition, molecular orientation 


\section{Introduction}

Polymerization of oriented mono- and multi-layer structures containing diacetylene functionality has produced an assortment of colored, robust, highly oriented, and environmentally responsive films (1). These two-dimensional polydiacetylene films, where the conjugation runs parallel to the film surface, have been prepared as Langmuir monolayers (2), Langmuir-Blodgett (LB) multilayer films (3), bilayer systems (4), and self-assembled monolayers (SAM) (5). Numerous applications for these films have been explored, including biomimetic surfaces for protein studies (6), biosensors (7), NLO materials (8), and biomineralization templates (9).

These materials exhibit high structural order in the many different forms yielding colored films that range from blue to red. The color of each material is a product of the conformation of the conjugated backbone. This conformation is affected by the orientation and packing of the hydrocarbon side chains pendant to the backbone that can either alter the length or change the mode of $\pi$-conjugation (10). A variety of analytical techniques, such as electron diffraction (11), FT-IR (12), resonance Raman (13), and fluorescence polarization (14), have been employed to identify the molecular orientation of the side chains and backbones.

Recently, atomic force microscopy (AFM) has been used to image polydiacetylene films at the nanometer scale to evaluate film structure based on topography (15), friction anisotropy (16), and surface features $(12,17,18)$. Although the technique is quite powerful, the images reveal a wide range of film quality. In the past, LB films of polydiacetylenes were prepared using metal ion salts (e.g., $\mathrm{CdCl}_{2}, \mathrm{CaCl}_{2}$ ) to stabilize the films prior to deposition. For monolayer deposition, a metal ion salt was essential to obtain quality films of amphiphiles with carboxylic acid headgroups 
$(3,18,19)$. The use of metal ion salts, however, can complicate the structures produced either altering molecular orientation through the metal ion chelation $(14,20)$ or depositing as salt crystals in or on the LB film.

In this paper, we will describe the formation of mono- and trilayer films of polydiacetylenes polymerized at the air-water interface on a pure water subphase. The blue or red films are subsequently transferred onto mica, silicon, or glass substrates using a horizontal deposition technique to capture innate features in the polymerized films. A carboxylic acid functionalized amphiphile, 10,12-pentacosadiynoic acid (I), unstable on a pure water subphase as a monolayer, forms a stable trilayer that is readily polymerized. and deposited onto substrates. Stable polydiacetylene monolayer structures could be prepared in the absence of salts using an ethanolamido functionalized analog of I, N-(2ethanol)-10,12-pentacosadiynamide (II). The amide functionality should provide some stabilization of the monolayer through lateral intermolecular hydrogen bonded networks at the headgroup position. AFM images of the deposited films reveal highly oriented, linear features that extend for tens to hundreds of micrometers within a discrete domain for both monolayer and trilayer films.

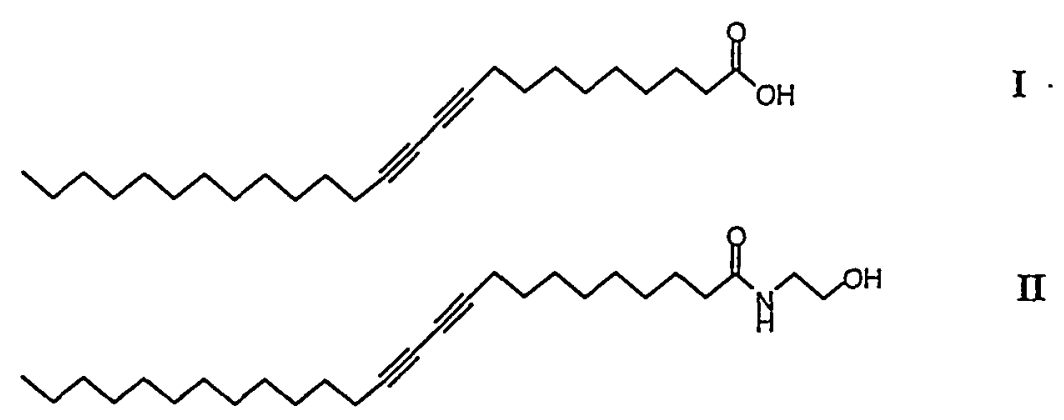




\section{Materials and Methods}

All solvents for purification and synthesis were of reagent grade (Fisher) and used as received. Solvents for monolayer spreading were of spectroscopic grade. The monolayer subphase was pure water (resistivity $\geq 18 \mathrm{M} \Omega-\mathrm{cm}$ ) filtered through a Barnstead Nanopure system (Dubuque, IA). NMR analyses were performed on a Bruker AMX 400 (Fremont, CA). Infrared absorption spectra were taken on a Perkin-Elmer 1750 FTIR Spectrometer (Norwalk, CT). UV-vis spectral data were collected on a Perkin-Elmer Lambda 19 Spectrophotometer. Elemental analyses were performed by Desert Analytics (Tucson, AZ).

10,12-Pentacosadiynoic acid (I) was obtained from Farchan/GFS Chemicals (Powell, $\mathrm{OH}$ ) as a bluish powder. The powder was taken up in a small amount of ethylacetate and passed through a silica gel column with $25 \%$ ethylacetate/hexanes $\left(R_{f}=\right.$ 0.23). The appropriate fractions were collected and evaporated to dryness below room temperature to minimize polymerization of the diacetylene monomer. A white powder was isolated and stored at $-20^{\circ} \mathrm{C}$, protected from light.

$\mathrm{N}$-(2-ethanol)-10,12-pentacosadiynamide (II). To a solution of I (2.50 g, 6.67 mmole) in $\mathrm{CH}_{2} \mathrm{Cl}_{2}(50 \mathrm{~mL})$ was added oxalyl chloride $(0.64 \mathrm{~mL}, 0.93 \mathrm{~g}, 7.3 \mathrm{mmole})$ in dropwise manner. The initial gas evolution subsided after one hour of stirring, then a drop of DMF was added to complete the reaction. Following another hour of stirring, the solution was evaporated to dryness and the residuals dissolved in a mixture of THF (40 $\mathrm{mL}$ ) and ethanolamine (1.61 $\mathrm{mL}, 1.63 \mathrm{~g}, 26.7 \mathrm{mmole})$. The mixture was stirred overnight. Ethylacetate $(60 \mathrm{~mL})$ and $\mathrm{H}_{2} \mathrm{O}(60 \mathrm{~mL})$ were then added to the reaction 
mixture, the mixture shaken, layers separated, and the aqueous layer extracted with fresh ethylacetate $(2 \times 60 \mathrm{~mL})$. The organics were combined and washed with $\mathrm{H}_{2} \mathrm{O}(60 \mathrm{~mL})$, followed by aqueous saturated $\mathrm{NaHCO}_{3}(60 \mathrm{~mL})$, and then with brine $(60 \mathrm{~mL})$. The organic layer was isolated and further dried over anhydrous $\mathrm{MgSO}_{4}$, filtered, then concentrated in vacuo. The residuals were flash column chromatographed on silica gel (ethylacetate, $R_{f}=0.18$ ). II was isolated as a white powder. Over time the compound developed a slight blue tinge even in storage at $-20^{\circ} \mathrm{C}$, protected from light. All spectral and elemental analysis data were identical with that reported in the literature (21).

All $\pi$-A isotherms and Langmuir film preparations were performed on a Nima 2011 circular Langmuir trough (Coventry, England). The trough was situated on a vibration isolation table inside a class 100 clean room. The pure water subphase was kept at a temperature of $15 \pm 0.2^{\circ} \mathrm{C}$. All diacetylene monomers were spread on the water surface in a 50\% chloroform/benzene solution. While solutions of I were completely homogeneous, solutions of II needed to be passed through a 0.2 micron filter to remove small traces of red polymer then diluted to volume. All films were incubated for $10-15$ minutes at zero pressure prior to compression. The compression rate for the $\pi-\mathrm{A}$ isotherms and for LB preparation was $100 \mathrm{~cm}^{2} / \mathrm{min}$ ( $4 \AA^{2} /$ molecule $\left.\bullet \mathrm{min}\right)$.

For UV polymerization at the air-water interface, the Langmuir films were first compressed to a surface pressure of $20 \mathrm{mN} / \mathrm{m}$, then equilibrated at that pressure for 20 30 minutes. Within the first $5-10$ minutes the films stabilized to a constant molecular area. UV irradiation of the compressed films was performed with two Oriel pen lamps laterally spaced $8 \mathrm{~cm}$ apart. Their combined incident power output directly below the center of the lamps at various distances are shown in Table 1. The lamps were set to 
specific heights above the air-water interface and the films irradiated for fixed time periods (Table 1) to yield specific color transitions. After the lamps were turned off the films sat undisturbed for a few minutes, then the water was slowly drained off by aspiration. The monolayers were laid down on mica (freshly cleaved), silicon (piranhacleaned), or glass substrates that were presubmerged in the aqueous subphase before monolayer spreading. After the monolayer was securely deposited on the substrate, the substrate was removed by forceps and allowed to dry in the cleanroom at a tilted angle.

A Nanoscope IIIA AFM (Digital Instruments, Santa Barbara, CA) operating in contact mode was used to obtain topographic images. Measurements with the AFM were acquired under laboratory ambient conditions. The scan rate was $3 \mathrm{~Hz}(=$ lines $/ \mathrm{sec})$ unless otherwise noted. Silicon nitride cantilevers (Digital Instruments, Santa Barbara, CA) with a nominal normal force constant of $0.06 \mathrm{~N} / \mathrm{m}$ were used for all measurements.

Microscopic (far-field) sample fluorescence was recorded using a Leitz optical fluorescence microscope equipped with dichroic beam filters and polarized white light from a Xenon lamp. The sample was illuminated with 520-550 nm light, and emission wavelengths greater than $590 \mathrm{~nm}$ were passed to a CCD camera, which captured the field of view for the images.

\section{Results and Discussion}

Amphiphiles of I and II on pure water produced the pressure-area $(\pi-\mathrm{A})$ isotherms shown in Figure 1. Both films had identical take-off areas of $25 \AA^{2} /$ molecule, corresponding to the molecular cross-section of the hydrocarbon-diacetylene structure. The film of I collapses at low pressure $(\sim 12 \mathrm{mN} / \mathrm{m})$, but upon over-compression reaches 
a stable solid phase with a limiting molecular area of $\sim 8 \AA^{2} /$ molecule. This overcompressed state corresponds to a stable trilayer structure (20). The film of $\mathbf{I}$ was stable as the monolayer with a collapse pressure of ca. $35 \mathrm{mN} / \mathrm{m}$ and an extrapolated molecular area at zero pressure of $25 \AA^{2} /$ molecule.

\section{Insert Figure 1}

Both diacetylene films were polymerized at the air-water interface and deposited onto solid substrates using the horizontal deposition method described below. Films equilibrated at $20 \mathrm{mN} / \mathrm{m}$ were polymerized to the blue-phase by exposure to incidence powers of $40 \mu \mathrm{W} / \mathrm{cm}^{2}$ for I and $23 \mu \mathrm{W} / \mathrm{cm}^{2}$ for II over a period of 30 seconds (Table 1). Red-phase films were produced by exposing the trilayer of $\mathrm{I}$ to $500 \mu \mathrm{W} / \mathrm{cm}^{2}$ and the monolayer of II to $40 \mu \mathrm{W} / \mathrm{cm}^{2}$ for 5 minutes.

\section{Insert Table 1}

Upon UV exposure the diacetylene films not only changed color but also contracted with increasing exposure times (Table 1). Both blue-phase films of I and II contracted only slightly in area by $5 \%$ and $2 \%$, respectively. The red-phase films, on the other hand, dramatically contract by $32 \%$ for I and $15 \%$ for $\mathrm{II}$. The contraction observed for $\mathrm{II}$ is consistent with that observed for similar diacetylene monolayers in the literature $(3,14,20)$, however, the behavior of the I trilayer is unique. Figures $2 \mathrm{~A}$ and $2 \mathrm{~B}$ shows the area vs. time plots for mono- and trilayer films of $I I$ and $I$, respectively, prior to and 
during a 5 minute UV exposure. Both films reach stable structures at a constant surface pressure of $20 \mathrm{mN} / \mathrm{m}$ prior to UV irradiation. Immediately following the onset of UV irradiation, the films began to contract. Interestingly, in the first 30 seconds of UV irradiation both films reach a pseudo-stable blue-phase structure, where the molecular area shows signs of leveling off (dotted arrows in Figure 2A and 2B). With further exposure the films continue to contract while progressively converting to the red form. Within the first 3 minutes of exposure more than $90 \%$ of the total contraction has occurred. After 4 minutes, the films have reached stable structures. All polymerizations were conducted in clean-room air and no expansion of the monolayer was observed at any time before, during, or after UV exposure (20).

\section{Insert Figure 2}

The polymerized films were then transferred onto solid substrates for UV-vis spectral measurements, polarized fluorescence microscopy, and AFM imaging. Initially, we attempted to deposit the films using a vertical dipping LB deposition technique. However, the very rigid nature of the films, especially in the blue-phase, prevented any reasonable film transfer. Typically, for the blue films of both I and II, only a few small $\left(<1 \mathrm{~cm}^{2}\right)$ film fragments would break off and transfer onto the substrate $(4 \times 2.5 \mathrm{~cm})$. Because of the crystalline-like structure of the blue films, the total area would remain constant at a pressure of $20 \mathrm{mN} / \mathrm{m}$, but a visible hole in the film would persist where the transfer took place. Unlike the homogeneously continuous and stiff blue-phase films, the red-phase films had visible cracks throughout. This allowed the film to compress with 
vertical deposition so that film transfer was significantly improved. However, even in this case the transfer was less than $70 \%$ and the film exhibited oriented features induced from the transfer process.

To capture the innate features of the polymerized films on the pure water subphase, as well as achieving high transfer ratios, a horizontal deposition method was employed. Substrates were situated on a support at a depth of $1-2 \mathrm{~mm}$ below the airwater interface and oriented plane-parallel to the surface. Following UV exposure, the film was transferred onto the solid substrate by lowering the water level by aspiration. Once the film draped over the substrate, excess film was removed from the outside edges of the substrate using the aspirator. The substrate was removed and the trapped water between the film and substrate was allowed to evaporate in clean-room air. Visual inspection indicated no macroscopic changes in the transferred blue- and red-phase films relative to their appearance on the air-water interface.

UV-vis spectral data of the polydiacetylene films were typical of blue and redphase forms. Figure 3 displays the absorption spectra of poly(I) trilayer films after 30 seconds at $40 \mu \mathrm{W} / \mathrm{cm}^{2}$ (blue-phase) and one after a 5 minute exposure at $500 \mu \mathrm{W} / \mathrm{cm}^{2}$ (red-phase). Poly(II) monolayer films gave nearly identical spectra although with about $70 \%$ less intensity, as would be expected for a sample one-third the thickness of the poly(I) trilayer. The absorption spectra are somewhat broad indicating inhomogeneous polymerization within the film. Although the transferred films were situated directly below the pen lamps to enhance exposure uniformity, the inhomogeneous polymerization most likely results from residual non-uniform UV exposure in the set up. 
We are currently developing UV exposure systems that will produce uniform illumination over large areas.

\section{Insert Figure 3}

AFM images of the blue- and red-phase forms of poly(I) and poly(II) on mica are shown in Figure 4. The coverage for all films was nearly uniform for the entire mica substrate. The topographic images in Figure 4 are taken of areas with cracks or defects in the film to allow for some contrast. Over $95 \%$ of the transferred film was atomically flat with up to $100 \mu \mathrm{m}$ crystalline domains observed. Films produced on silicon substrates with both native and thermally grown oxide possess the same quality.

\section{Insert Figure 4}

The choice of a pure water subphase was a result of our initial attempts to prepare mono- and multilayer films using metal ion salts of $\mathrm{I}$. $\mathrm{CdCl}_{2}$ and $\mathrm{CaCl}_{2}$ salts, at concentrations from $1 \mu \mathrm{M}$ to $3 \mathrm{mM}$, were used in the subphase to prepare stable monolayers of I. Following both vertical and horizontal deposition, AFM images revealed sub-micron size particles, most probably salt crystals, dispersed throughout the samples. An example of such an image is shown in Figure 5. It was clear that such contaminated films would not be amenable to any molecular level studies not only because of the surface roughness produced by the crystallites, but also from their long and/or short range effects on the chromatic molecular actuation of the film (15). 


\section{Insert Figure 5}

The $50 \mu \mathrm{m}$ scale images of Figure 4 show overall polydiacetylene morphology and film structure. For the blue-phase film of $\mathrm{I}$, square edges and straight-line cracks are observed giving the film a crystalline-like appearance (Figure 4A). The edges of the redphase film of Figure 4B are not as straight nor are any square edges observed. However, polarized fluorescence microscopy of the red films has shown that the polydiacetylene backbone direction in a single domain was highly oriented (15). By setting the analyzing polarizers at $90^{\circ}$ to the backbone direction the emission of a given trilayer domain could be completely extinguished. This confirms that the conjugated backbones within each domain are highly ordered, and that there is structural registry between the three layers of the red film. In other words, the backbones are oriented along the same direction in all three layers within a domain. Thus, the crystallinity of the domains exists not only parallel to the substrate, but normal to it as well. Similarly, high polarization contrast was also observed for fluorescence from the red-phase film of II, indicating that the backbones are also highly oriented in the monolayer.

AFM imaging could also resolve structural differences between the blue- and redphase films of both I and II. Distinct height differences between the blue- and red-phase trilayer stacks of poly $(\mathrm{I})$ were measured at $7.4 \pm 0.8$ and $9.0 \pm 0.9 \mathrm{~nm}$, respectively. Similarly, the blue- and red-phase poly(II) monolayer films exhibited proportional height differences of $2.7 \pm 0.3$ and $3.1 \pm 0.3 \mathrm{~nm}$, respectively. The poly(II) films, like the poly(I) films, were crystalline in appearance (Figures 4C and 4D) with nearly 
homogeneous coverage over the mica substrate with few cracks or multilayer stacks observed.

Figure 6 shows higher resolution views of the blue-phase films for I and II revealing the molecular orientation and highly regular structures within the materials. We have previously determined that the striated patterns observed in Figure 6 run parallel to the polydiacetylene backbone $(15,16)$. Each defined line feature is $10-20 \mathrm{~nm}$ wide indicating that a set of $20-40$ polymers creates a single feature. The height contrast is $\sim 0.2 \mathrm{~nm}$. The origin of these features is not fully understood. It is possible that they arise from slightly different packing densities of groups of neighboring backbones. In any event, this topographic contrast clearly reveals the highly oriented nature of the backbones within each domain. With such highly oriented films, near molecular level structures can be readily and reproducibly imaged. The high quality of these films has allowed us to observe dramatic properties such as nanometer scale conversion from blue to red by mechanical stress (15) and strong friction anisotropy (16).

\section{Insert Figure 6}

These results provide insight into the possible molecular orientation of diacetylene films as they convert to blue- and red-phase polymer structures. The headgroup interactions and alkyldiyne chain stacking should dominate the film structure of the monomeric diacetylene Langmuir films. The ability of the amide headgroup of II to form intermolecular hydrogen bonded structures (Figure 7), similar to $\beta$-sheets in proteins, may explain the stability of this monolayer film on pure water. I films on pure 
water subphase, on the other hand, are unstable as monolayers but stack favorably into trilayer structures. Carboxylic acid dimer formation may aid in stabilizing the structure (Figure 7). Indeed, stable bilayer islands are commonly observed on top of the I trilayer. The high registry of the diacetylene packing permits rapid topochemical polymerization of the diyne monomers to the ene-yne conjugation upon UV illumination resulting in the blue-phase polydiacetylene. Little change in the amphiphile packing, and thus little reorientation of the alkyl sidechains occurs, as evidenced by the minimal contraction of the film (Table 1). However, the hybridization change from $\mathrm{sp}$ to $\mathrm{sp}^{2}$ for the terminal alkyne carbons creates a stress on the polymer as a result of the $180^{\circ}$ to $120^{\circ}$ bond angle conversion (22). Apparently, this stress is eventually relieved by the reorientation of the alkyl side chains to a more vertical orientation resulting in height increases in both the poly(I) and poly (II) films, closer packing (Figure 2 and Table1), and change in $\pi$-conjugation that produces the red-form polymer (12). We are currently investigating the alkyl side chain reorientation by coupling molecular modeling with experimental studies to further understand the color transitions and modes of actuation of these films.

\section{Insert Figure 7}

\section{Conclusion}

Langmuir films of I and $\boldsymbol{I}$ polymerized at the air-water interface produced excellent samples for molecular level imaging with AFM. The simple procedure described to produce polydiacetylene films on a pure water subphase eliminated 
contamination problems associated with salt formation in the deposited film. Due to the stiffness of the polymerized films, we were able to use a horizontal deposition technique to yield solid supported films that were similar to their state on the water surface. The photochromic conversion of the diacetylenes to their blue and red forms showed a dramatic change in molecular orientation that was evident from the changes in molecular area during polymerization and in the AFM topologic images and height measurements.

\section{Acknowledgements}

We would like to thank Dr. Mary Crawford for the power measurement readings of the UV pen lamps, and Dr. Seema Singh for the AFM image of Figure 5. RWC acknowledges the support of the Natural Sciences and Engineering Research Council of

Canada. Sandia is a multiprogram laboratory operated by Sandia Corporation, a Lockheed Martin Company, for the United States Department of Energy under Contract DE-AC04-94AL85000.

To whom correspondence should be addressed. Fax (505)844-5470. E-mail: dvsasak@sandia.gov

$\S$ Present address: Dept. of Engineering Physics, University of Wisconsin-Madison, 1500 Engineering Dr., Madison, WI, 53706. 


\section{References}

1. Bloor, D. and Chance, R.R. "Polydiacetylenes" Martinus Nijhoff Publishers, Boston, 1985.

2. Wilson, T.E. and Bednarski, M.D. Langmuir 8, 2361 (1992).

3. Kuriyama, K., Kikuchi, H., and Kajiyama, T. Langmuir 12, 6468 (1996).

4. Charych, D.H., Nagy, J.O., Spevak, W., and Bednarski, M.D. Science 261, 585 (1993).

5. Kim, T., Chan, K.C., and Crooks, R.M. J. Am. Chem. Soc. 119, 189 (1997).

6. Bader, H., van Wagenen, R., Andrade, J.D., and Ringsdorf, H. J. Coll. Interf. Sci. 101, 246 (1984).

7. Charych, D. and Nagy, J.O. Chemtech 24 (1996).

8. Sarkar, A., Okada, S., Nakanishi, H., and Matsuda, H. Macromolecules 31, 9174 (1998).

9. Litvin, A.L., Samuelson, L.A., Charych, D.H., Spevak, W., and Kaplan, D.L. J. Phys. Chem. 99, 12065 (1995).

10. Rughooputh, S. D. D. V., Bloor, D., Phillips, D., Jankowiak, R., Schütz, L., and Bässler, H. Chem. Phys. 125, 355 (1988).

11. Day, D. and Lando, J.B. Macromolecules 13, 1483 (1980).

12. Lio, A., Reichert, A., Ahn, D. J., Nagy, J. O., Salmeron, M., and Charych, D. H., Langmuir 13, 6524 (1997).

13. Saito, A., Urai, Y., and Itoh, K. Langmuir 12, 3938 (1996).

14. Miyano, K. and Mori, A. Thin Solid Films 168, 141 (1989).

15. Carpick, R. W., Sasaki, D. Y., and Burns, A. R., Langmuir in press. 
16. Carpick, R. W., Sasaki, D. Y., and Burns, A. R., Tribology Lett. in press

17. Marti, O., Ribi, H. O., Drake, B., Albrecht, T. R., Quate, C. F., and Hansma, P. K., Science 239, 50 (1988).

18. Sheth, S.R. and Leckband, D.E. Langmuir 13, 5652 (1997).

19. Collins, M. J. Poly. Sci.: Part B: Polym. Phys. 26, 367 (1988).

20. Day, D. and Ringsdorf, H. J. Polym. Sci.: Poly. Lett. 16, 205 (1977).

21. Wilson, T.E., Spevak, W., Charych, D.H., and Bednarski, M.D. Langmuir 10, 1512 (1994).

22. Menzel, H., Mowery, M.D., Cai, M., and Evans, C.E. J. Phys. Chem. B 102, 9550 (1998). 
Table 1. UV Polymerization of Diacetylene Films at the Air-Water Interface ${ }^{a}$

\begin{tabular}{|c|c|c|c|c|c|}
\hline Amphiphile & Exposure time & $\begin{array}{c}\text { Lamp height } \\
(\mathrm{cm})\end{array}$ & $\begin{array}{c}\text { Incident Power } \\
\left(\mu \mathrm{W} / \mathrm{cm}^{2}\right)\end{array}$ & Film color & $\begin{array}{c}\% \text { Area } \\
\text { contraction }\end{array}$ \\
\hline I & $30 \mathrm{sec}$ & 10 & 40 & Blue & $5 \%$ \\
I & $5 \mathrm{~min}$ & 6 & 500 & Red & $32 \%$ \\
II & $30 \mathrm{sec}$ & 15 & 23 & Blue & $2 \%$ \\
II & $5 \mathrm{~min}$ & 10 & 40 & Red & $15 \%$ \\
\hline
\end{tabular}

a) At $20 \mathrm{mN} / \mathrm{m}$ surface pressure on a pure water subphase maintained at $15^{\circ} \mathrm{C}$.

b) Determined using Model 365 exposure analyzer (Optical Associates Inc.)energy, with a maximum absorption at $260 \mathrm{~nm}$. 


\section{Figure Captions}

Figure 1. Pressure-area ( $\pi-\mathrm{A})$ isotherms of $\mathrm{I}(--)$ and $\mathbf{I}(-)$ diacetylene compounds on a pure water subphase at $15^{\circ} \mathrm{C}$.

Figure 2. Plots of molecular area vs. time of II (A) and I (B) films maintained at a surface pressure of $20 \mathrm{mN} / \mathrm{m}$ on a pure water subphase at $15^{\circ} \mathrm{C}$, prior to and during a 5 minute UV irradiation exposure. Dotted arrows indicate the point at which pseudo-stable blue-phase structures exists. The time axis shown is a portion of the total curve that begins from the film compression followed by pressure equilibration and finally UV exposure.

Figure 3. UV-vis absorption spectra of blue-phase (B) and red-phase (R) poly(I) trilayer films deposited on glass substrates.

Figure 4. $50 \mu \mathrm{m}$ scale topographic AFM images of poly(I) in the blue- (A) and red-phase (B) and poly(II) in the blue- (C) and red-phase (D) on mica.

Figure 5. $50 \mu \mathrm{m}$ scale topographic AFM image of horizontally deposited poly(I) monolayer from an aqueous $1 \mathrm{mM} \mathrm{CdCl}{ }_{2}$ subphase on mica. Numerous sub-micron scale crystallites (white dots) cover the surface. 
Figure 6. High resolution $1 \mu \mathrm{m}$ scale topographic AFM image of blue-phase poly(I) (A) and poly(II) (B) showing highly oriented film structure (on mica). The height contrast of the striations is $0.2 \mathrm{~nm}$.

Figure 7. Schematic of molecular orientation of $I I$ and its subsequent conversion to poly(II) upon UV irradiation (above). A hydrogen bonded network at the headgroup position is drawn. Below is a illustration of poly(I) in its trilayer form. 


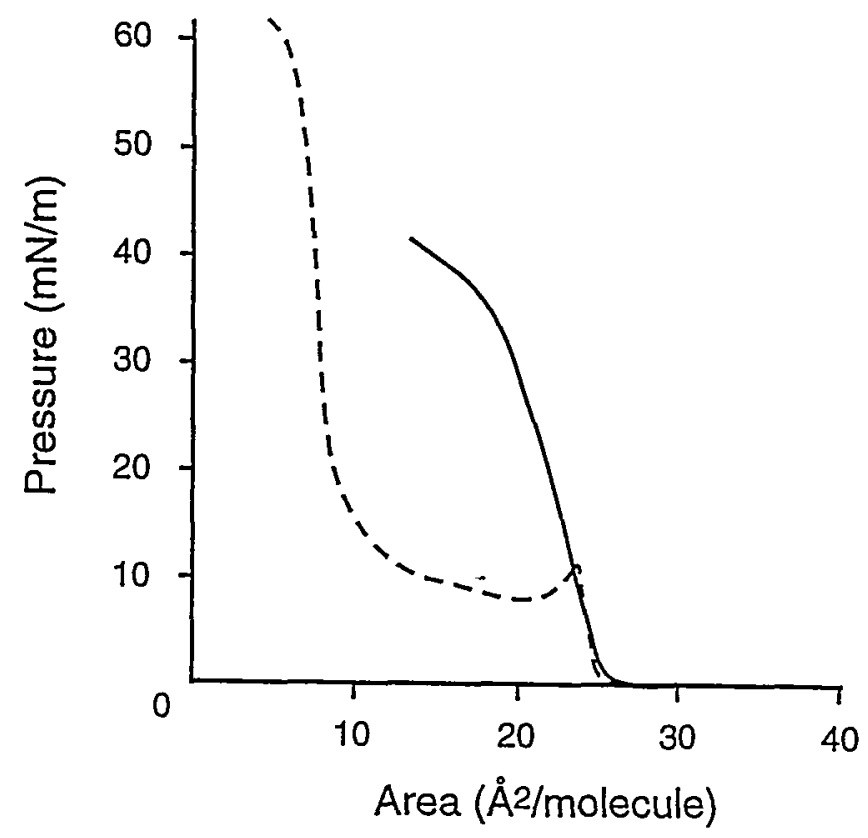

Figure 1

High Molecular Orientation in Mono- and Tri-Layer Polydiacetylene Films Imaged by Atomic Force Microscopy

D. Y. Sasaki, R. W. Carpick, and A. R. Burns 

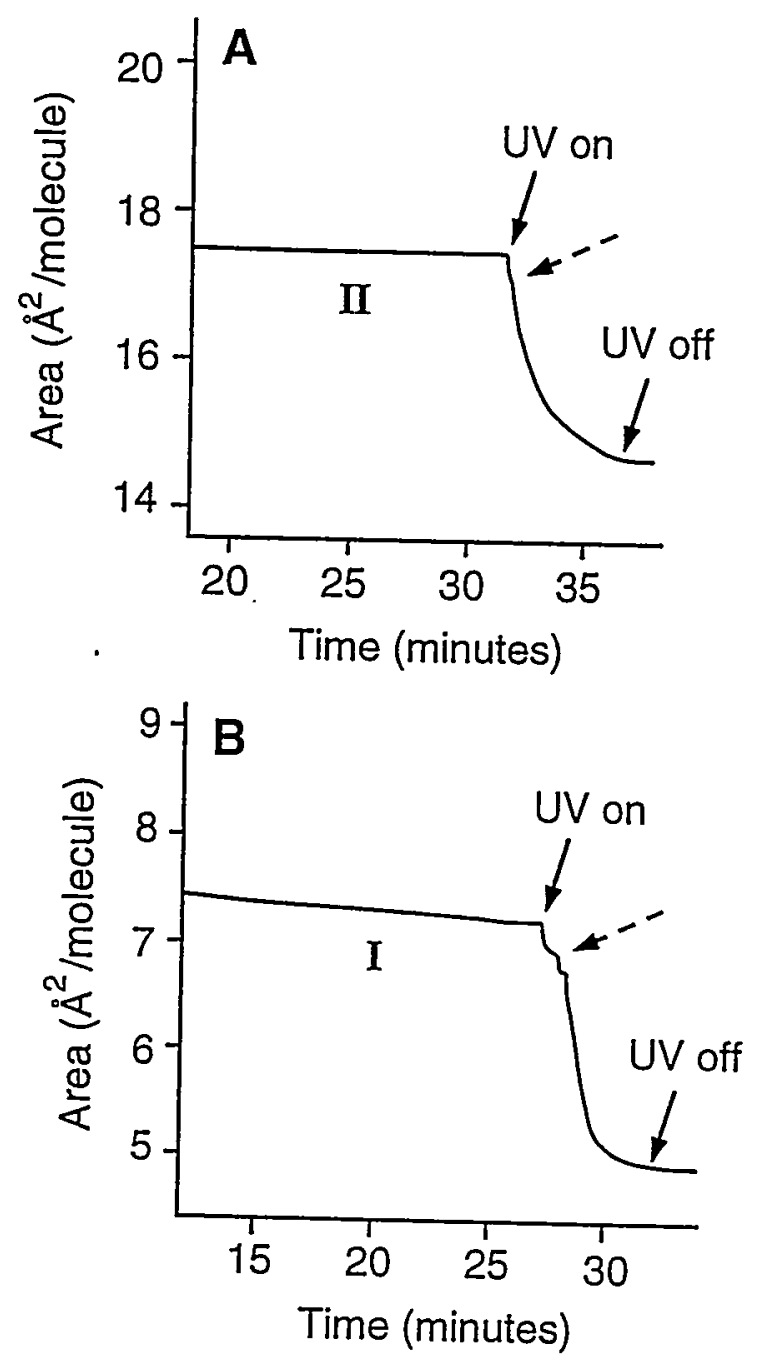

Figure 2

High Molecular Orientation in Mono- and Tri-Layer Polydiacetylene Films Imaged by Atomic Force Microscopy

D. Y. Sasaki, R. W. Carpick, and A. R. Burns 


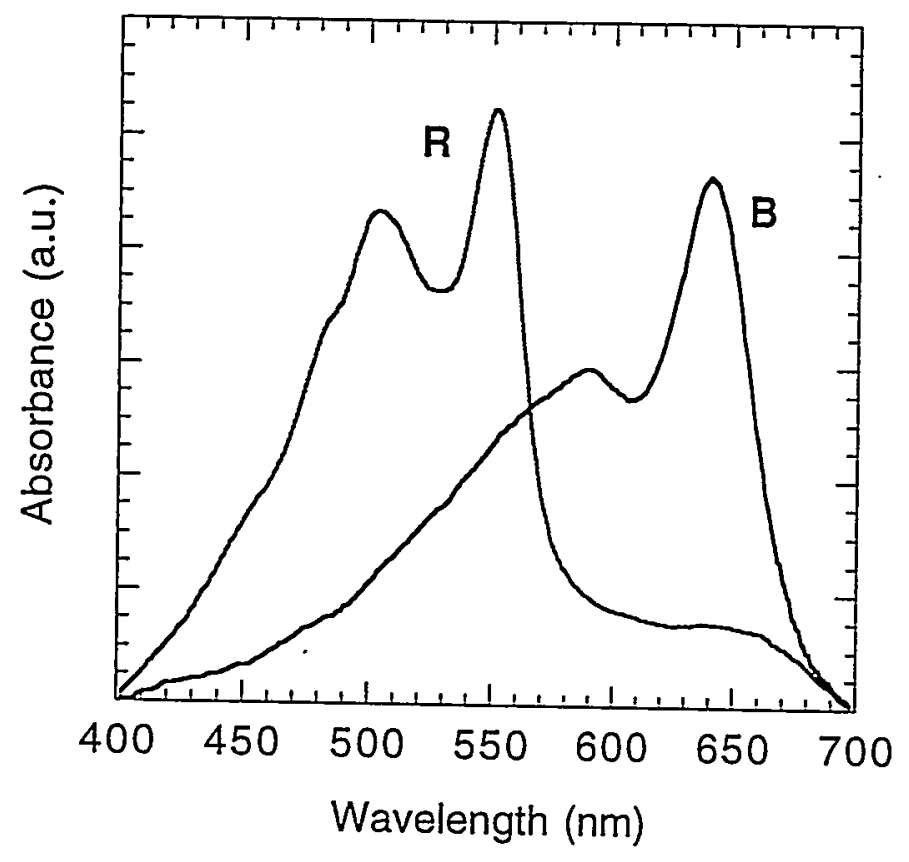

Figure 3

High Molecular Orientation in Mono- and Tri-Layer Polydiacetylene Films Imaged by Atomic Force Microscopy

D. Y. Sasaki, R. W. Carpick, and A. R. Burns 

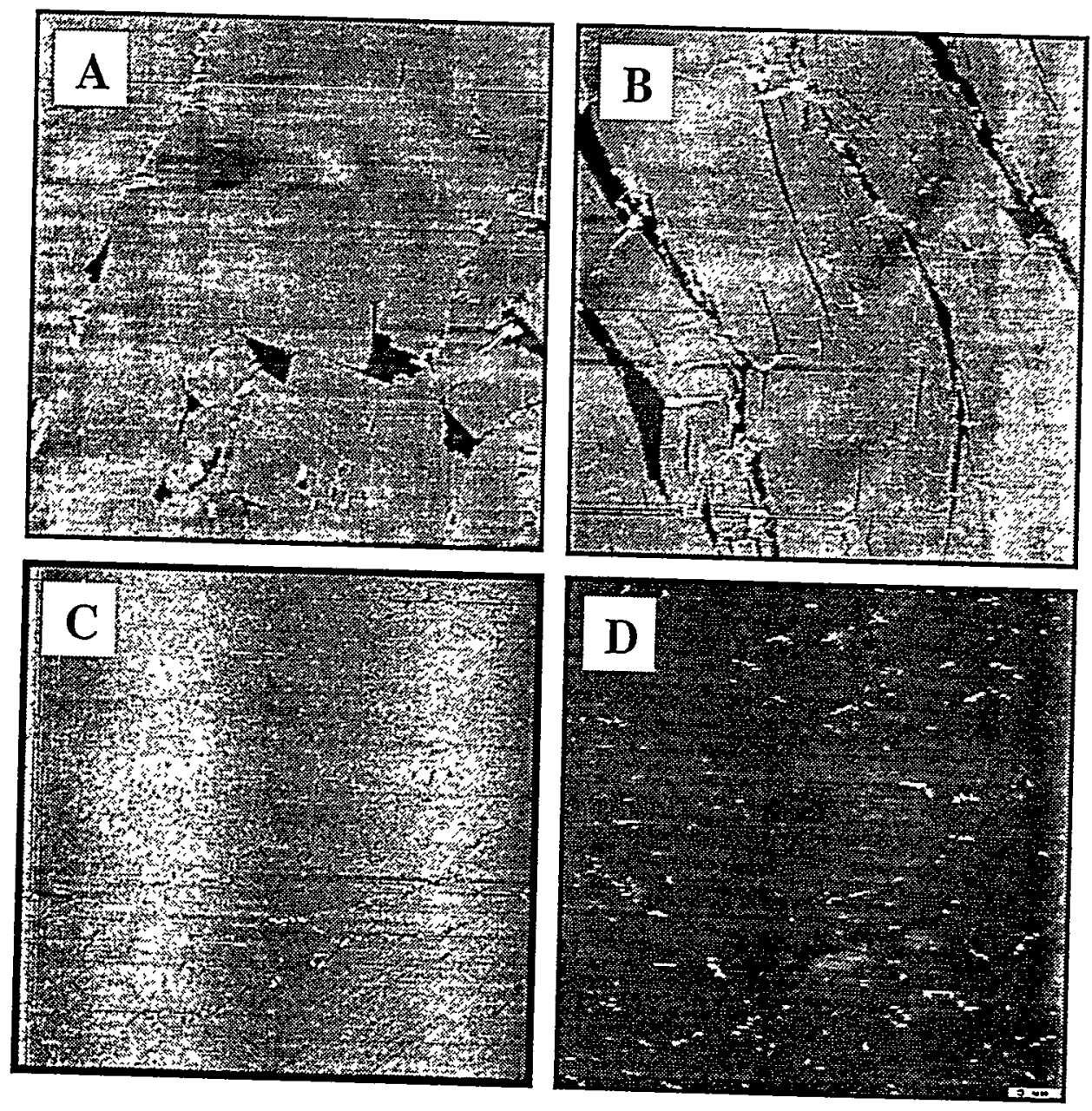

Figure 4

High Molecular Orientation in Mono- and Tri-Layer Polydiacetylene Films Imaged by Atomic Force Microscopy

D. Y. Sasaki, R. W. Carpick, and A. R. Burns 


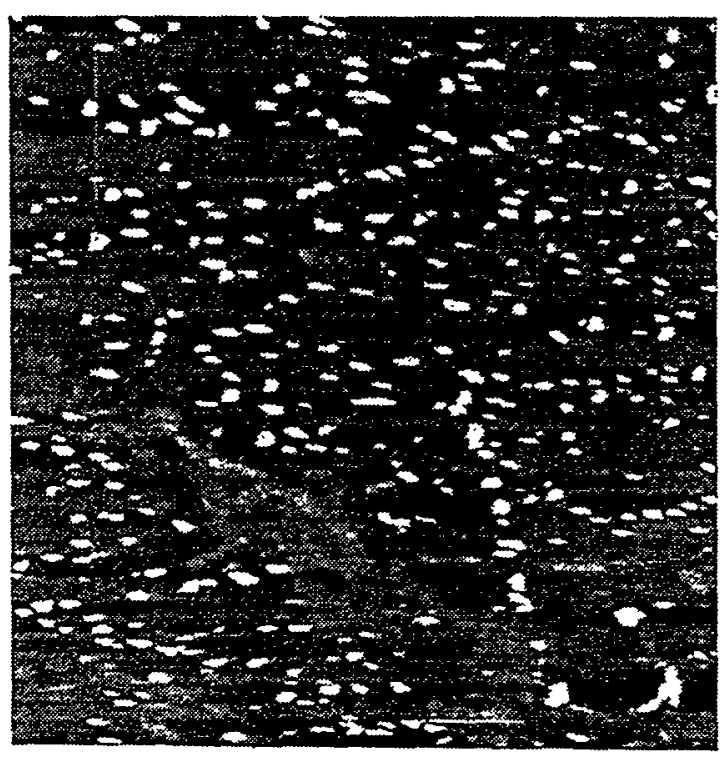

Figure 5

High Molecular Orientation in Mono- and Tri-Layer Polydiacetylene Films Imaged by Atomic Force Microscopy D. Y. Sasaki, R. W. Carpick, and A. R. Burns 

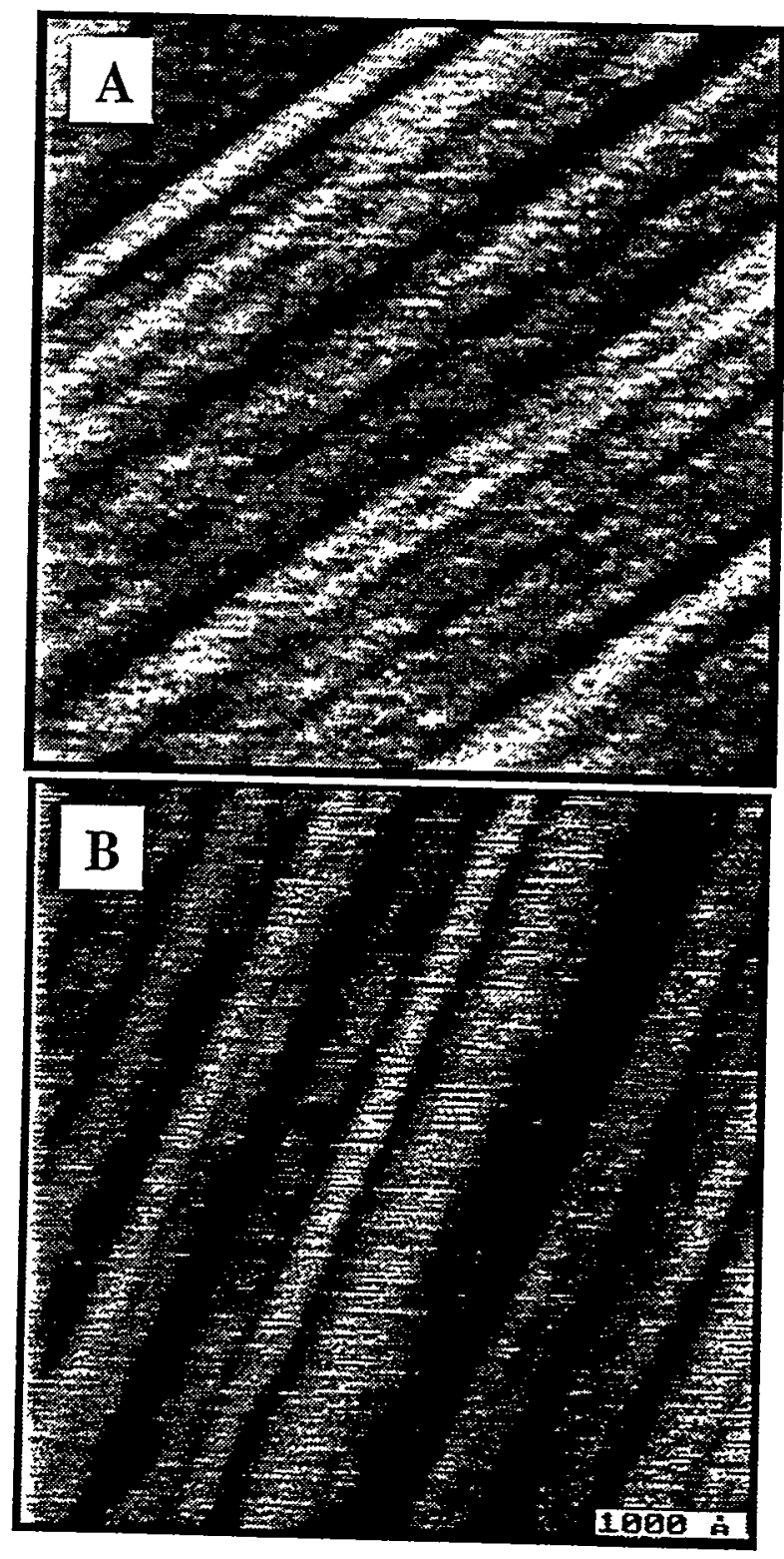

Figure 6

High Molecular Orientation in Mono- and Tri-Layer Polydiacetylene Films Imaged by Atomic Force Microscopy

D. Y. Sasaki, R. W. Carpick, and A. R. Burns 

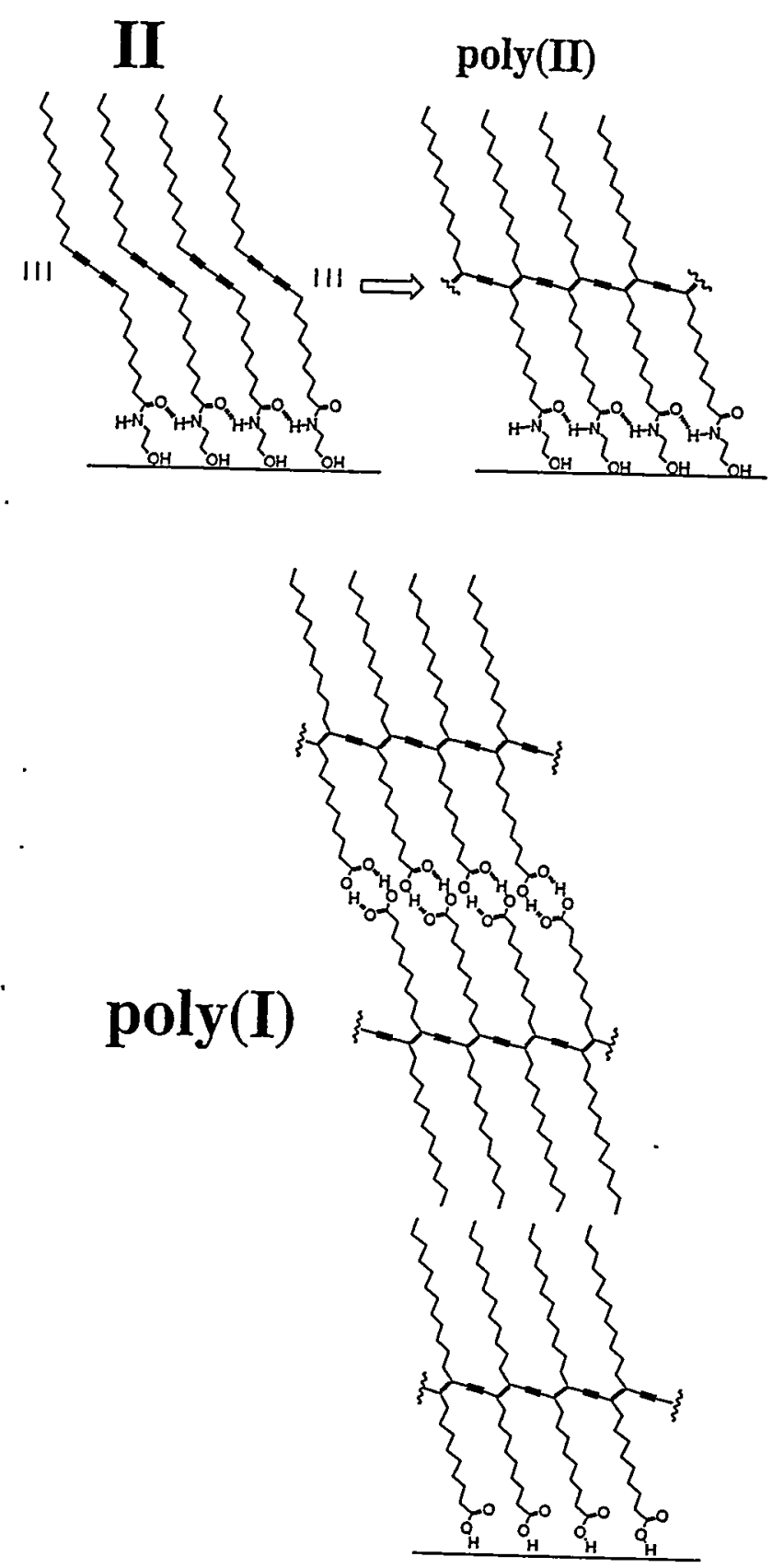

Figure 7

High Molecular Orientation in Mono- and Tri-Layer Polydiacetylene Films Imaged by Atomic Force Microscopy

D. Y. Sasaki, R. W. Carpick, and A. R. Burns 